\title{
ON UNITARY EQUIVALENCE OF ARBITRARY MATRICES $\left({ }^{1}\right)$
}

\author{
BY \\ HEYDAR RADJAVI
}

1. Introduction. The problem we wish to study is that of deciding whether two given square matrices $A$ and $B$ over the field of complex numbers are unitarily equivalent, i.e., whether there exists a unitary matrix $U$ such that $B=U^{-1} A U$. This decision can be made easily if a computable set of canonical forms for all matrices is obtained, that is, if there exists an algorithm which associates with any given matrix $A$ another matrix $C(A)$ such that if $A$ and $B$ are two matrices and $C(A)$ and $C(B)$ their respective forms obtained by the algorithm, then $C(A)$ is equal to $C(B)$ if and only if $A$ and $B$ are unitarily equivalent. The solution of this problem for the set of normal matrices is well known; the canonical set consists of all diagonal matrices with complex entries arranged in some order agreed on. We shall make use of facts concerning the diagonalization of normal matrices.

The analog of the present problem, where similarity is considered instead of unitary equivalence is much simpler (Jordan canonical forms). We cannot expect as simple a canonical set in the case of unitary equivalence. The following example shows how much vaster the set of canonical forms in this case can be as compared to the set of Jordan canonical forms:

Let $n>2$. Take all $n \times n$ matrices of the form

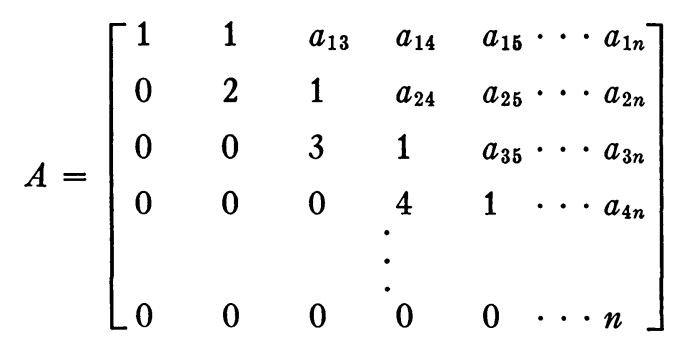

where the $a_{i j}$ are complex numbers. Under similarity all of these matrices are equivalent and their common Jordan form is

$$
\operatorname{Diag}(1,2,3, \cdots, n) \text {; }
$$

but under unitary transformations two matrices of the above type are equiv-

Received by the editors July 17, 1961.

(1) Acknowledgment. This paper is a condensation of a Master's Thesis at the University of Minnesota. The author wishes to acknowledge his indebtedness to Professor G. K. Kalisch for his encouragement in the preparation of this dissertation and to the National Science Foundation (Grant G 14137) for financial support. 
alent if and only if they are equal. This means that to just one Jordan canonical form there corresponds an uncountable number of canonical forms under unitary equivalence; in fact each $A$ of the above-mentioned form is its own canonical form if we require that canonical forms be triangular with eigenvalues arranged in ascending order, and the $(i, i+1)$-elements be positive.

The present problem was considered by J. Brenner [1]. On the basis of Brenner's work, D. E. Littlewood made further remarks [2]. A special case was considered by B. E. Mitchell [3]. Another attack on this problem is contained in a dissertaion by Vincent V. McRae [5]. The method which we shall use in this paper will enable us to find canonical forms for matrices $A$ not only under the full group of unitary transformations $\{U\}$, but also under certain subgroups of this group which we call "direct groups." It is the reduction of equivalence under the full unitary group to that under such direct subgroups which provides the fundamental idea involved in Brenner's work [1] and also in the present paper. This reduction is carried out in a stepwise manner to successively "finer" direct groups. Our work differs from Brenner's [1] in that he sketches a double induction based on diagonalizing a block $B$ of the matrix $A$ by multiplying it by unitary blocks $U$ and $V$ on the left and on the right respectively and considering commutators, while in considering a block $B$ of $A$, we separate out the effect of multiplying $B$ on the left by $U$ from that of multiplying by $V$ on the right. This avoids a great deal of manipulation and permits us to describe more tightly how decisions on unitary equivalence of two matrices can be made in a finite number of steps, and also how to establish canonical forms. In addition, the method used in this paper yields some intermediate results interesting in themselves (such as Theorem 1), and also considers simultaneous unitary equivalence of ordered sets of matrices.

2. Preliminary remarks and definitions. By the norm of a column vector or a row vector $X$ with components $\left(a_{1}, a_{2}, \cdots, a_{n}\right)$ will be meant the nonnegative square root of the quantity $\left|a_{1}\right|^{2}+\left|a_{2}\right|^{2}+\cdots+\left|a_{n}\right|^{2}$; it will be denoted by $\|X\|$. By a vector we shall always mean a column vector. The symbol $A^{*}$ will denote the conjugate transpose of the matrix $A$, so that if $X$ is a vector, then $\|X\|^{2}=X^{*} X$. If a matrix $A$ is partitioned into blocks $A_{i j}$, we shall refer to the arrangement

$$
A_{11}, A_{12}, A_{13}, \cdots ; A_{21}, A_{22}, A_{23}, \cdots ; A_{31}, A_{32}, A_{33}, \cdots ; \cdots
$$

of the $A_{i j}$ as the natural ordering of the blocks.

Definition. If $H$ is a subgroup of the group of $n \times n$ unitary matrices, we say two matrices $A$ and $B$ are equivalent under $H$ if $B=U^{*} A U$ for some member $U$ of $H$.

Definition. Consider the set $G$ of all $n \times n$ unitary matrices of the form

$$
U=\operatorname{Diag}\left(U_{1}, U_{2}, \cdots, U_{m}\right) \text {, }
$$


where $U_{i}$ is any $r_{i} \times r_{i}$ unitary matrix and where $r_{1}+r_{2}+\cdots+r_{m}=n$. Then $G$ is a subgroup of the group of $n \times n$ unitary matrices and will be called an unrestricted direct group. The sequence of integers $\left\{r_{i}\right\}$ is called the size sequence of $G$, or, for brevity, the size of $G$.

Definition. We shall make use of subgroups of unitary matrices which are more restricted than those given in the preceding definition: We consider the unrestricted direct group $G$ and let

$$
E_{1}, E_{2}, \cdots, E_{s}
$$

be a partition of the set of integers $\{1,2, \cdots, m\}$ into $s$ disjoint subsets. Let $H$ be the set of all members

$$
U=\operatorname{Diag}\left(U_{1}, U_{2}, \cdots, U_{m}\right)
$$

of $G$ with the property that $U_{i}=U_{j}$ whenever $i$ and $j$ belong to the same subset $E_{k}$. Then $H$ is a subgroup of $G$ and will be called a direct group; the sequences $\left\{r_{i}\right\}$ and $\left\{E_{j}\right\}$ will be called the size and the partition of $H$. (If the integers $i$ and $j$ belong to the same set $E_{k}$, then $r_{i}$ and $r_{j}$ are necessarily equal.)

Definition. If $U=\operatorname{Diag}\left(U_{1}, U_{2}, \cdots, U_{m}\right)$, then $U_{j}$ will be called the $j$ th component of $U$.

Definition. Let $H$ be a direct group of size $\left\{r_{i}\right\}$ and partition $\left\{E_{j}\right\}$. Then a typical member of $H$ is of the form

$$
U=\operatorname{Diag}\left(U_{1}, U_{2}, \cdots, U_{k}, \cdots, U_{m}\right) .
$$

Let $E_{t}$ be that member of $\left\{E_{j}\right\}$ which contains the integer $k$. Let $G_{0}$ be an unrestricted direct group of $r_{k} \times r_{k}$ unitary matrices with size $\left\{p_{i}\right\}, p_{1}+p_{2}$ $+\cdots+p_{e}=r_{k}$. In the expansion (1) of $U$ replace $U_{k}$ and all other $U_{i}$ with $i$ in $E_{t}$ by

$$
V=\operatorname{Diag}\left(V_{1}, V_{2}, \cdots, V_{e}\right),
$$

a typical member of $G_{0}$. The set of all unitary matrices $U$ thus obtained from the members of $H$ forms a subgroup $K$ of $H$ which is itself a direct group; it is called the refinement of $H$ by $G_{0}$ in the kth place. The direct group $K$ is uniquely determined by $H, G_{0}$, and the integer $k$.

NoTE. Unrestricted direct groups are direct groups whose corresponding partitions consist of subsets $E_{i}$ each of which has only one element. We shall omit the adjective "unrestricted" when no confusion is caused by doing so.

Definition. Let $H$ be a direct group with size $\left\{r_{i}\right\}$ and partition $\left\{E_{j}\right\}$, $i=1,2, \cdots, m ; j=1,2, \cdots, n$. Let the integers $e$ and $f$ be in $E_{p}$ and in $E_{q}$ respectively and assume that $r_{e}=r_{f}$. Consider the new partition of the set of integers $\{1,2, \cdots, m\}$ which is obtained from the partition $\left\{E_{j}\right\}$ by uniting $E_{p}$ and $E_{q}$. Call this new partition $\left\{F_{k}\right\}$-after relabelling the sets. The direct group $K$ with size $\left\{r_{i}\right\}$ and partition $\left\{F_{k}\right\}$ will be called a restriction of $H$, or more precisely an $(e, f)$-restriction of $H$. 
We now present Propositions 1, 2, and 3 which are the key to the method used in this paper.

\section{Three propositions.}

Proposition 1. Let $B$ be an $r \times s$ matrix. Then there exists an $r \times r$ unitary matrix $U$ such that $U B$ has mutually orthogonal row vectors $X_{1}, X_{2}, \cdots, X_{r}$ with $\left\|X_{1}\right\| \geqq\left\|X_{2}\right\| \geqq \cdots \geqq\left\|X_{r}\right\|$. Furthermore, there exists a unique direct group $H$ of $r \times r$ unitary matrices, completely determined by $B$, such that the set of all unitary matrices $U$ which have the above-stated property is precisely the coset $H U_{0}$, where $U_{0}$ is any unitary matrix having the property.

Proof. Consider $B B^{*}$ which is an $r \times r$ nonnegative-definite Hermitian matrix. There exists a unitary matrix $U$ which transforms $B B^{*}$ into its diagonal form

$$
U\left(B B^{*}\right) U^{*}=\operatorname{Diag}\left(c_{1}, c_{2}, \cdots, c_{r}\right)
$$

with $c_{1} \geqq c_{2} \geqq \cdots \cdots c_{r} \geqq 0$. In general, the $c_{i}$ are not all distinct. Let the first $r_{i}$ of the $c_{i}$ be equal, then the next $r_{2}$ of them equal but distinct from the first $r_{1}$, and so on. This gives rise to integers $\left\{r_{i}\right\}$ with $r_{1}+r_{2}+\cdots+r_{m}=r$. Let $H$ be the unrestricted direct group with size $\left\{r_{i}\right\} . H$ is uniquely determined by $B B^{*}$ and, furthermore, the set of all unitary matrices that diagonalize $B B^{*}$ with diagonal elements in descending order, is precisely the coset $H U$.

Next observe that the $(i, j)$-element of $U B B^{*} U=(U B)(U B)^{*}$ is $X_{i} X_{j}^{*}$, where the $X_{i}$ are the row vectors of $U B$. It is now easily verified that the matrix $U$ and the direct group $H$ have the properties required in the statement of the proposition.

Definition. If the row vectors of an $r \times s$ matrix $B$ are mutually orthogonal and all have the same norm, then $B$ is called a row-orthogonal matrix. A matrix $B$ is row-orthogonal if and only if $B B^{*}$ is a nonnegative multiple of the identity matrix. For future purposes we also define column-orthogonal matrices in an analogous manner. A matrix $B$ is column-orthogonal if and only if $B^{*} B$ is a nonnegative multiple of the identity.

Proposition 2. Let $B$ be an $r \times s$ matrix and $V$ any $s \times s$ unitary matrix. Then $B$ and $B V$ give rise to the same direct group $H$ and the same coset $H U_{0}$ as occurred in Proposition 1.

The proof follows from the equation $(B V)(B V)^{*}=B B^{*}$.

Considering $B^{*} B$ instead of $B B^{*}$ we can prove

Proposition 3. Let $B$ be an $r \times s$ matrix. Then there exists an $s \times s$ unitary matrix $U$ such that $B U$ has mutually orthogonal column vectors $X_{1}, X_{2}, \cdots, X_{r}$ with $\left\|X_{1}\right\| \geqq\left\|X_{2}\right\| \geqq \cdots \geqq\left\|X_{r}\right\|$. Furthermore, there exists a unique direct group $H$ of $s \times s$ unitary matrices, completely determined by $B$, such that the set of all unitary matrices having the above property is precisely the coset $U H$. 
The column analog of Proposition 2 also holds.

Definition. The matrix $U$ of Proposition 1 is called $a$ row-fixer of $B$ and the direct group $H$, the row-fixed group of $B$. A column-fixer of $B$ and the column-fixed group of $B$ are defined similarly in connection with Proposition 3.

\section{A series of algorithms.}

Algorithm 1. Let $H$ be a direct group of $n \times n$ matrices with size $\left\{r_{i}\right\}$ and partition $\left\{E_{j}\right\}$ and let $A$ be an $n \times n$ matrix. Partition $A$ into blocks $A_{i j}$ conforming with $H$, i.e., such that $A_{i j}$ is an $r_{i} \times r_{j}$ matrix. Assume that at least one of the $A_{i j}$ is not row-orthogonal, and let $A_{r s}$ be the first $A_{i j}$ in the natural order which is not row-orthogonal. Apply Proposition 1 to $A_{r s}$ and let $U_{1}$ be a row-fixer and $G$ the row-fixed group of $A_{r s}$. Let $E_{k}$ be that member of $\left\{E_{j}\right\}$ which contains $r$. Let $U$ be that member of $H$ whose $i$ th component is $U_{1}^{*}$ whenever $i$ is in $E_{k}$ and whose remaining components are all identity matrices. We shall call $A_{1}=U^{*} A U$ a transform of $A$ under $A$ lgorithm 1. The refinement $H_{1}$ of $H$ by $G$ in the $r$ th place will be called the refinement of $H$ induced by Algorithm 1 on $A$.

Proposition 4. Two matrices $A$ and $B$ are equivalent under a direct group $H$ if and only if they give rise to the same refinement $H_{1}$ of $H$ under Algorithm 1 and their transforms $A_{1}$ and $B_{1}$ are equivalent under $H_{1}$.

Proof. Assume $A$ and $B$ are equivalent under $H$. Partition $A$ and $B$ into blocks $A_{i j}$ and $B_{i j}$ conforming with $H$. Then $B_{i j}=V_{i}^{*} A_{i j} V_{j}$, where the $V_{k}$ are fixed unitary matrices and $V_{i}=V_{j}$ if $i$ and $j$ belong to the same set in the partition of $H$. Since the row-orthogonality of one of the two matrices $A_{i j}$ and $B_{i j}$ implies that of the other, the first non-row-orthogonal blocks of $A$ and $B$ occur at the same position $(r, s)$. It follows from the above propositions that $A_{r s}$ and $B_{r s}$ have the same fixed group $G$ and hence $A$ and $B$ give rise to the same refinement $H_{1}$ of $H$. Next let $U_{1}$ and $W_{1}$ be any row-fixers of $A_{r s}$ and $B_{r s}$ respectively; then, by Proposition 2, there exists a member $V$ of $G$ such that $W_{1} V_{r}^{*}=V U_{1}$. Let $A_{1}$ and $B_{1}$ be the transforms of $A$ and $B$ under Algorithm 1 obtained by making use of $U_{1}$ and $W_{1}$. Then the $(i, j)$-blocks $A_{i j}^{\prime}$ and $B_{i j}^{\prime}$ of $A_{1}$ and $B_{1}$ are given below in all possible cases. Letting $E_{p}$ denote the set containing the integer $r$ in the partition of $H$, then:

(1) if $i \notin E_{p}$ and $j \notin E_{p}$, then $A_{i j}^{\prime}=A_{i j}$ and $B_{i j}^{\prime}=B_{i j}=V_{i}^{*} A_{i j} V_{j}=V_{i}^{*} A_{i j}^{\prime} V_{j}$;

(2) if $i \in E_{p}$ and $j \notin E_{p}$, then $A_{i j}^{\prime}=U_{1} A_{i j}$ and $B_{i j}^{\prime}=W_{1} B_{i j}=W_{1} V_{r}^{*} A_{i j} V_{j}$ $=V U_{1} A_{i j} V_{j}=V A_{i j}^{\prime} V_{j}$;

(3) if $i \notin E_{p}$ and $j \in E_{p}$, then $A_{i j}^{\prime}=A_{i j} U_{1}^{*}$ and $B_{i j}^{\prime}=B_{i j} W_{1}^{*}=V_{i}^{*} A_{i j} V_{r} W_{1}^{*}$ $=V_{i}^{*} A_{i j}\left(W_{1} V_{r}^{*}\right)^{*}=V_{i}^{*} A_{i j}^{\prime} V^{*} ;$ and

(4) if $i \in E_{p}$ and $j \in E_{p}$, then $A_{i j}^{\prime}=U_{1} A_{i j} U_{1}^{*}$ and $B_{i j}^{\prime}=W_{1} B_{i j} W_{1}^{*}$ $=W_{1} V_{r}^{*} A_{i j} V_{r} W_{1}^{*}=V U_{1} A_{i j} U_{1}^{*} V=V A_{i j}^{\prime} V^{*}$.

It follows that $B_{1}=S^{*} A_{1} S$, where $S$ is obtained from $\operatorname{Diag}\left(V_{i}\right)$ by replacing $V_{j}$ by $V$ whenever $j \in E_{p}$, but, since $V$ belongs to $G$, this means that $A_{1}$ 
and $B_{1}$ are equivalent under $H_{1}$, the refinement of $H$ by $G$ in the $r$ th place. This completes the proof of Proposition 4 in one direction. The proof of the converse is trivial.

Algorithm 2. Let $H$ be a direct group and $A$ a matrix partitioned into blocks conforming with $H, A_{r s}$ be the first $A_{i j}$ in the natural order which is not column-orthogonal. Apply Proposition 3 to $A_{r s}$ and let $U_{1}$ be a column-fixer and $G$ the column-fixed group of $A_{r s}$. Let $E_{k}$ be that member of $H$ whose $i$ th component is $U_{1}$ whenever $i$ is in $E_{k}$ and whose other components are all identity matrices. The refinement $H_{1}$ of $H$ by $G$ in the sth place will be called the refinement of $H$ induced by Algorithm 2 on $A$, and $A_{1}=U^{*} A U a$ transform of $A$ under Algorithm 2. The analog of Proposition 4 holds.

We now state a theorem which we consider to be of some interest in itself.

TheOREM 1. Let $H$ be a direct group of $n \times n$ unitary matrices. There exists an algorithm, called Algorithm 4 in the sequel, which, when applied to any $n \times n$ matrix $A$, associates with it a unique direct subgroup $H(A)$ of $H$ and a matrix $A_{0}$ equivalent to $A$ under $H$ such that

(1) $A_{0}$ when partitioned into blocks conforming with $H(A)$ has the form

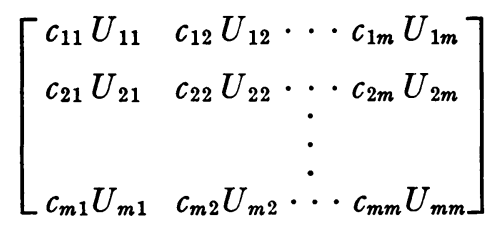

where the $c_{i j}$ are nonnegative real numbers and the $U_{i j}$ unitary matrices $\left.{ }^{2}\right)$ and

(8) a matrix $B$ is equivalent to $A$ under $H$ if and only if $H(A)=H(B)$ and $B_{0}$ is equivalent to $A_{0}$ under $H(A)$.

The proof will follow the description of Algorithm 4.

Algorithm 3. Let $A_{i j}$ be the blocks of $A$ conforming with $H$. If every $A_{i j}$ is row-orthogonal, put

$$
A^{(1)}=A, \quad H^{(1)}=H
$$

otherwise apply Algorithm 1 to $A$; let $A_{1}$ be the transform of $A$ under Algorithm 1 and $H_{1}$ the corresponding refinement of $H$. Repeat the process, i.e., if every block of $A_{1}$ when partitioned into blocks conforming with $H_{1}$ is roworthogonal, put

$$
A^{(1)}=A_{1}, \quad H^{(1)}=H_{1} ;
$$

otherwise apply Algorithm 1 with the new group $H_{1}$, let $A_{2}$ be the transform of $H_{1}$, etc. Thus we obtain a sequence of direct groups $\left\{H_{i}\right\}$, where $H_{i+1}$ is a refinement of $H_{i}$. Each time that Algorithm 1 is applicable, a proper refine-

${ }^{(2)}$ The $c_{i j}$ corresponding to nonsquare blocks are, therefore, necessarily zero. 
ment of the preceding direct group is obtained, so that after a finite number of steps we find a direct group $H_{m}$ and a matrix $A_{m}$, where in the partition of $A_{m}$ conforming with $H_{m}$ every block is row-orthogonal. Then let

$$
A^{(1)}=A_{m} \quad \text { and } \quad H^{(1)}=H_{m} .
$$

Clearly $H^{(1)}$ is uniquely determined by this process, and furthermore $A$ and $B$ are equivalent under $H$ if and only if they give rise to the same $H^{(1)}$ and $A^{(1)}$ and $B^{(1)}$ are equivalent under $H^{(1)}$. (This follows from repeated applications of Proposition 4).

Next consider $A^{(1)}$ and $H^{(1)}$ and repeat the process described above with Algorithm 2 instead of Algorithm 1. This will, after a finite number of steps, give rise to a matrix $A^{(2)}$ and a unique direct group $H^{(2)}$ such that $A^{(2)}$, when partitioned into blocks conforming with $H^{(2)}$, has only columnorthogonal blocks. Again, two matrices $A$ and $B$ are equivalent under $H$ if and only if they give rise to the same direct group $H^{(2)}$ and $A^{(2)}$ is equivalent to $B^{(2)}$ under $H^{(2)}$. We will call $H^{(2)}$ the subgroup of $H$ induced by Algorithm 3 on $A$. The matrix $A^{(2)}$ will be called the transform of $A$ under Algorithm 3.

Algorithm 4. Let $H$ be a direct group. Find the transform $A^{(2)}$ of $A$ under Algorithm 3 and the corresponding induced subgroup $H^{(2)}$ of $H$. Having found $A^{(2 i)}$ and $H^{(2 i)}$ apply Algorithm 3 with the new direct group $H^{(2 i)}$ and let $A^{(2 i+2)}$ be the transform of $A^{(2 i)}$ and $H^{(2 i+2)}$ the corresponding induced subgroup of $H^{(2 i)}$. Each time that $H^{(2 i+2)}$ is not equal to $H^{(2 i)}$, it has at least one more component than $H^{(2 i+2)}$; hence after a finite number of steps we will have $A^{(2 m+2)}=A^{(2 m)}$ and $H^{(2 m+2)}=H^{(2 m)}$. Let $A_{0}=A^{(2 m)}$ and $H_{0}=H(A)$ $=H^{(2 m)}$. The subgroup $H^{(2 m)}$ of $H$ is uniquely determined by Algorithm 4 and $A$ is equivalent to $B$ under $H$ if and only if $H_{0}=H(A)=H(B)$ and $A_{0}$ is equivalent to $B_{0}$ under $H_{0}$.

Proof of Theorem 1. Observe that if $A_{0}$ and $H_{0}$ are the matrix and direct group obtained by Algorithm 4, then in the partition of $A_{0}$ into blocks conforming with $H_{0}$, the blocks are both row-orthogonal and column-orthogonal and, therefore, nonnegative multiples of unitary matrices.

Algorithm 5. Let $H$ be a direct group of $n \times n$ unitary matrices with size $\left\{r_{i}\right\}$ and partition $\left\{E_{j}\right\}$. Let $A$ be an $n \times n$ matrix whose blocks in the partition conforming with $H$ are nonnegative multiples $c_{i j} U_{i j}$ of unitary matrices $U_{i j}$. If for each nonzero $c_{i j}$ the integers $i$ and $j$ belong to the same set $E_{k}$, let $H_{*}=H$ and $A_{*}=A$; otherwise let $c_{r s}$ be the first nonzero $c_{i j}$ in the natural order for which $i$ and $j$ do not belong to the same set. Let $r$ be in $E_{f}$ and $s$ in $E_{g}$. Let $U$ be that member of $H$ whose $i$ th component is $U_{r s}$ for all $i$ in $E_{f}$ and whose remaining components are identity matrices. Put $A_{*}=U^{*} A U$ and let $H_{*}$ be the $(f, g)$-restriction of $H$.

Proposition 5. If the matrix $A_{*}$ is obtained from $A$ by Algorithm 5 and if $H_{*}$ is the corresponding restriction of $H$ as in Algorithm 5 , then the $(r, s)$-block of $U^{*} A_{*} U$ in the partition conforming with $H_{*}$ is $c_{r 8} I$, where $I$ is the identity 
matrix, for all $U$ in $H_{*}$; furthermore, two matrices $A$ and $B$ are equivalent under $H$ if and only if they give rise to the same direct group $H_{*}$ under Algorithm 5 and $A_{*}$ is equivalent to $B_{*}$ under $H_{*}$.

Proof. That the $(r, s)$-block of $U^{*} A_{*} U$ is equal to $c_{r s} I$ for all $U$ in $H_{*}$ is easy to see. If $A$ and $B$ are equivalent under $H$, they have the same ordered set of $c_{i j}$, so that the integers $r$ and $s$ are the same for $A$ and $B$; hence they give rise to the same restriction $H_{*}$ of $H$. The $(r, s)$-block of $B_{*}$ is also $c_{r s} I$ and it follows at once that $A_{*}$ and $B_{*}$ are equivalent under $H_{*}$. The proof of the converse is trivial.

Algorithm 6. Let $H$ be a direct group of $n \times n$ matrices. Let $A$ be an $n \times n$ matrix whose blocks in the partition conforming with $H$ are nonnegative multiples of unitary matrices. Apply Algorithm 5 to $A$ with the group $H$ and obtain $A_{*}$ and $H_{*}$; next apply Algorithm 5 to $A_{*}$ with the new group $H_{*}$ and obtain $A_{* *}=A_{(2)}$ and $H_{* *}=H_{(2)}$. Continue this process, i.e., having found $A_{(k)}$ and $H_{(k)}$, apply Algorithm 5 to $A_{(k)}$, with the direct group $H_{(k)}$ and obtain $A_{(k+1)}$ and $H_{(k+1)}$. Each time that Algorithm 5 is applicable nontrivially, the number of sets in the partition $\left\{E_{k}\right\}$ of the direct group decreases by 1 ; hence after a finite number of steps we obtain $A_{(m+1)}=A_{(m)}$ and $H_{(m+1)}=H_{(m)}$. We shall call $A_{(m)}$ a transform of $A$ under Algorithm 6 and $H_{(m)}$ the subgroup of $H$ induced by Algorithm 6 on $A$. The following proposition is an immediate consequence of the construction of $A_{(m)}$ and $H_{(m)}$.

Proposition 6. Assume $A$, when partitioned to conform with $H$, has blocks which are nonnegative multiples of unitary matrices. Let $A^{\prime}$ be a transform of $A$ under Algorithm 6, and $H^{\prime}$ the corresponding induced subgroup of $H$. Let $\left\{E_{k}\right\}$ be the partition of $H^{\prime}$. Let $A_{i j}^{\prime}$ be the blocks of $A^{\prime}$ when partitioned according to $H^{\prime}$. Then the $A_{i j}^{\prime}$ are nonnegative multiples $c_{i j} U_{i j}$ of unitary matrices $U_{i j}$ and $c_{i j}=0$ whenever $i$ and $j$ belong to distinct sets of the partition $\left\{E_{k}\right\}$.

Proposition 7. If two matrices $A$ and $B$ have nonnegative multiples of unitary matrices as blocks conforming with a direct group $H$, then they are equivalent under $H$ if and only if they give rise to the same induced subgroup $H^{\prime}$ of $H$ under Algorithm 6 and their transforms $A^{\prime}$ and $B^{\prime}$ under the algorithm are equivalent under $H^{\prime}$.

Proof. Apply Proposition 5 repeatedly.

Algorithm 7. Let $H$ be a direct group of $n \times n$ unitary matrices with partition $\left\{E_{k}\right\}$. Let $A$ be an $n \times n$ matrix whose blocks in the partition conforming with $H$ are nonnegative multiples $c_{i j} U_{i j}$ of unitary matrices $U_{i j}$, and such that $c_{i j}=0$ whenever $i$ and $j$ belong to two distinct sets in $\left\{E_{k}\right\}$. Then a member

$$
U=\operatorname{Diag}\left(U_{1}, \cdots, U_{m}\right)
$$

of $H$ transforms $A$ into $U^{*} A U$ whose blocks are $c_{i j} U_{i}^{*} U_{i j} U_{j}$, and by assump- 
tion $U_{i}=U_{j}$ if $c_{i j} \neq 0$. If for each nonzero $c_{i j}$, the matrix $U_{i j}$ is the identity matrix, put

$$
A^{\prime}=A, \quad H^{\prime}=H .
$$

Otherwise let $c_{r s}$ be the first nonzero number among the $c_{i j}$, in the natural order, for which $U_{i j}$ is not the identity matrix. Then there exists a unitary matrix $V_{1}$ such that $V_{1}^{*} U_{r s} V_{1}$ is diagonal; furthermore, if $m$ is the size of $U_{r s}$, there exists a unique direct group $G$ of $m \times m$ unitary matrices such that the set of unitary matrices that diagonalize $U_{r s}$ is precisely the coset $V_{i} G$. Let $U$ be that member of $H$ whose $i$ th component is $V_{1}$ whenever $i$ belongs to the set $E_{k}$ containing $r$, and whose remaining components are all identity matrices. Put $A^{\prime}=U^{*} A U$ and let $H^{\prime}$ be the refinement of $H$ by $G$ in the $r$ th place.

Proposition 8. If $H$ is a direct group and if $A$ and $B$ are two matrices of the form described in Algorithm 7, then $A$ and $B$ are equivalent under $H$ if and only if they give rise to the same refinement $H^{\prime}$ of $H$ under Algorithm 7 and their transforms $A^{\prime}$ and $B^{\prime}$ are equivalent under $H^{\prime}$.

The proof is similar to the proof of Proposition 4.

Definition. Let $H$ be a given direct group of $n \times n$ unitary matrices. Let $A$ be any $n \times n$ matrix. Apply Theorem 1 to $A$ with the direct group $H$ and let $H_{1}$ be the resulting subgroup of $H$ and $A_{1}$ the resulting matrix. Next apply Algorithm 6 to $A_{1}$ with the group $H_{1}$ and obtain $A_{2}$ and $H_{2}$. Then apply Algorithm 7 to $A_{2}$ with the group $H_{2}$ and let $H_{3}$ and $A_{3}$ be the resulting direct group and matrix respectively. We shall call $A_{3}$ a first reduced form of $A$ under $H$, and $H_{3}$ the first reduced subgroup of $H$ with respect to $A$.

Proposition 9. Any first reduced form of $A$ is equivalent to $A$ under $H$, and if $B$ is another matrix, then $B$ is equivalent to $A$ under $H$ if and only if $A$ and $B$ give rise to the same first reduced subgroup $H^{\prime}$ of $H$ and their first reduced forms under $H$ are equivalent under $H^{\prime}$.

The proof follows from Theorem 1 and the earlier propositions.

Algorithm 8. Let $H$ be a direct group of $n \times n$ unitary matrices. Given an $n \times n$ matrix $A$, find a first reduced form $A^{(1)}$ of $A$ under $H$ and the first reduced subgroup $H^{(1)}$ of $H$ with respect to $A$. Repeat the process, i.e., having found $A^{(j)}$ and $H^{(j)}$, let $A^{(j+1)}$ be a first reduced form of $A^{(j)}$ under $H^{(j)}$ and let $H^{(j+1)}$ be the first reduced subgroup of $H^{(j)}$ with respect to $A^{(j)}$. Each time that $H^{(j+1)} \neq H^{(j)}$, either $H^{(j+1)}$ has at least one more component than $H^{(j)}$ or the number of sets in the partition of $H^{(j+1)}$ is at least one less than that in the partition of $H^{(j)}$. Hence after a finite number of steps we obtain $H^{(k+1)}=H^{(k)}$. We shall call $H^{(k)}$ the reduced subgroup of $H$ with respect to $A$, and $A^{(m)}$ the reduced form of $A$. By this method the reduced subgroup $H^{(m)}$ of $H$ with respect to $A$ is uniquely determined and we have

Theorem 2. Let $H$ be a direct group of $n \times n$ unitary matrices and $A$ an 
$n \times n$ matrix. Let $H_{0}$ and $A_{0}$ be, respectively, the reduced subgroup of $H$ with respect to $A$ and the reduced form of $A$ under $H$. Then $A_{0}$ is invariant under $H_{0}$, i.e., $U^{*} A_{0} U=A_{0}$ for all members $U$ of $H_{0}$. Furthermore, two matrices $A$ and $B$ are equivalent under $H$ if and only if they give rise to the same reduced subgroup $H_{0}$ of $H$ and their reduced forms under $H$ are equal.

Proof. If for some member $U$ of $H_{0}$ we had $U^{*} A_{0} U \neq A_{0}$, then at least one of the Algorithms 4, 6, and 7 would be further applicable to $A_{0}$, which would contradict the construction of $A_{0}$ and $H_{0}$; hence $U^{*} A_{0} U=A_{0}$ for all $U$ in $H_{0}$. Repeated applications of Proposition 9 show that $A$ and $B$ are equivalent under $H$ if and only if they give rise to the same reduced subgroup $H_{0}$ of $H$ and their reduced forms $A_{0}$ and $B_{0}$ are equivalent under $H_{0}$, but it follows from the first part of this theorem that $B_{0}=U^{*} A_{0} U$ for some $U$ in $H_{0}$ if and only if $B_{0}=A_{0}$.

Thus we have, by Theorem 2, established canonical forms $A_{0}$ for all $n \times n$ matrices $A$ under any given direct group $H$ of $n \times n$ unitary matrices. These canonical forms are found by Algorithm 8 .

5. A related result. Theorem 2 also solves the problem of simultaneous unitary equivalence of two finite sets of matrices: Given two ordered sets of $n \times n$ matrices $\left\{A_{i}\right\}$ and $\left\{B_{i}\right\}, i=1,2, \cdots, m$ we are to decide whether or not there exists an $n \times n$ unitary matrix $U$ such that $B_{i}=U^{*} A_{i} U$ for all $i$. This problem is the same as that of deciding whether or not two $m n \times m n$ matrices

$$
A=\operatorname{Diag}\left(A_{1}, A_{2}, \cdots, A_{m}\right)
$$

and

$$
B=\operatorname{Diag}\left(B_{1}, B_{2}, \cdots, B_{m}\right)
$$

are equivalent under the direct group $H$ of $m n \times m n$ unitary matrices whose size is $\left\{r_{i}\right\}$ with $r_{i}=n, i=1,2, \cdots, m$ and whose partition consists of just one set containing all the integers $1,2, \cdots, m$. (The components of each member $U$ of $H$ are all equal.)

6. Triangular canonical forms under the full unitary group. The direct group $H$ of Theorem 2 can be taken to be the full group of $n \times n$ unitary matrices, and we may desire the canonical forms of matrices under unitary equivalence (i.e., under the full group of unitary matrices) to be triangular. This suggests the following procedure:

Triangularization. Let $A$ be an $n \times n$ matrix acting on an $n$-dimensional unitary space $V$ and let $e_{1}, e_{2}, \cdots, e_{n}$ be the eigenvalues of $A$ arranged in some order agreed on, where each eigenvalue is repeated as many times as its algebraic multiplicity requires. (By this is meant the multiplicity of the eigenvalue as a zero of the characteristic polynomial of $A$.) Consider the following subspaces of $H$ : 


$$
\begin{aligned}
& V_{1}=\left(A-e_{2} I\right)\left(A-e_{3} I\right) \cdots\left(A-e_{n} I\right) V, \\
& V_{2}=\left(A-e_{3} I\right)\left(a-e_{4} I\right) \cdots\left(A-e_{n} I\right) V, \\
& \dot{V}_{n-1}=\left(A-e_{n} I\right) V, \\
& V_{n}=V .
\end{aligned}
$$

Each $V_{i}$ is contained in $V_{i+1}$ for $i=1,2, \cdots, n-1$. Furthermore, $\left(A-e_{1} I\right) V_{1}$ $=0$ and $\left(A-e_{i} I\right) V_{i+1}=V_{i}$ for $i=1,2, \cdots, n-1$. Let $m_{i}$ be the dimension of $V_{i}$. Choose an orthonormal basis $\left\{X_{1}, X_{2}, \cdots, X_{n}\right\}$ for $V$ in which the first $m_{i}$ vectors lie in $V_{i}$ for $i=1,2, \cdots, n$. Letting $U=\left[X_{1}, X_{2}, \cdots, X_{n}\right]$, the matrix $A$ will take on the triangular form $A_{0}=U^{*} A U$ with respect to the new basis.

Let $r_{1}=m_{1}$ and $r_{i}=m_{i}-m_{i-1}$ for $i=2,3, \cdots, n$. Then $r_{i} \geqq 0$ for all $i$. Take the nonzero $r_{i}$ and relabel them to form the sequence $\left\{s_{i}\right\}$, where $s_{1}=r_{1}, s_{2}$ is the next nonzero $r_{i}$, and so on. Let $H$ be the unrestricted direct group of $n \times n$ unitary matrices with size $\left\{s_{i}\right\}$. We shall call $H$ the triangularizer of $A$. The eigenvalues, the numbers $m_{i}$ and therefore the $s_{i}$, are invariant under all unitary transformations. Hence, if two matrices $A$ and $B$ are triangularized by the above method giving rise to corresponding triangularizers $H_{A}$ and $H_{B}$, and to triangular forms $A_{0}$ and $B_{0}$, then $A$ is unitarily equivalent to $B$ if and only if $H_{A}=H_{B}$ and $A_{0}$ is equivalent to $B_{0}$ under $H_{A}$. Thus we have the following

Corollary to Theorem 2. Let $A_{0}$ be a triangular form of the matrix $A$ and let $H$ be its corresponding triangularizer. Let $C(A)$ be the reduced form of $A_{0}$ under $H$. Then the matrix $C(A)$ is the triangular canonical form of $A$ : Two matrices $A$ and $B$ are unitarily equivalent if and only if $C(A)=C(B)$.

\section{BiBLIOGRAPHY}

1. J. Brenner, The problem of unitary equivalence, Acta Math. 86 (1951), 297-308.

2. D. E. Littlewood, On unitary equivalence, J. London Math. Soc. 28 (1953), 314-322.

3. B. E. Mitchell, Unitary transformations, Canad. J. Math. 6 (1954), 69-72.

4. P. R. Halmos, Finite dimensional vector spaces, Van Nostrand, Princeton, N. J., 1958.

5. Vincent V. McRae, On the Unitary Similarity of matrices, Dissertation, The Catholic Univ. of America Press, Washington, D. C., 1955.

University of MinNesota, Minneapolis, Minnesota 